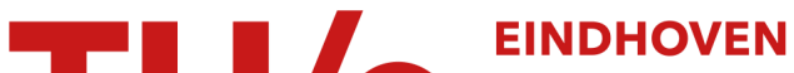 \\ UNIVERSITY OF \\ TECHNOLOGY
}

\section{Dynamics of the spatial electron density distribution of EUV- induced plasmas}

Citation for published version (APA):

van der Horst, R. M., Beckers, J., Osorio, E. A., \& Banine, V. Y. (2015). Dynamics of the spatial electron density distribution of EUV-induced plasmas. Journal of Physics D: Applied Physics, 48(43), [432001].

https://doi.org/10.1088/0022-3727/48/43/432001

\section{Document license:}

TAVERNE

DOI:

10.1088/0022-3727/48/43/432001

Document status and date:

Published: 04/11/2015

\section{Document Version:}

Publisher's PDF, also known as Version of Record (includes final page, issue and volume numbers)

\section{Please check the document version of this publication:}

- A submitted manuscript is the version of the article upon submission and before peer-review. There can be important differences between the submitted version and the official published version of record. People interested in the research are advised to contact the author for the final version of the publication, or visit the $\mathrm{DOI}$ to the publisher's website.

- The final author version and the galley proof are versions of the publication after peer review.

- The final published version features the final layout of the paper including the volume, issue and page numbers.

Link to publication

\section{General rights}

Copyright and moral rights for the publications made accessible in the public portal are retained by the authors and/or other copyright owners and it is a condition of accessing publications that users recognise and abide by the legal requirements associated with these rights.

- Users may download and print one copy of any publication from the public portal for the purpose of private study or research.

- You may not further distribute the material or use it for any profit-making activity or commercial gain

- You may freely distribute the URL identifying the publication in the public portal.

If the publication is distributed under the terms of Article $25 \mathrm{fa}$ of the Dutch Copyright Act, indicated by the "Taverne" license above, please follow below link for the End User Agreement:

www.tue.nl/taverne

Take down policy

If you believe that this document breaches copyright please contact us at:

openaccess@tue.nl

providing details and we will investigate your claim. 
Dynamics of the spatial electron density distribution of EUV-induced plasmas

This content has been downloaded from IOPscience. Please scroll down to see the full text.

2015 J. Phys. D: Appl. Phys. 48432001

(http://iopscience.iop.org/0022-3727/48/43/432001)

View the table of contents for this issue, or go to the journal homepage for more

Download details:

IP Address: 131.155.151.137

This content was downloaded on 08/12/2015 at 09:43

Please note that terms and conditions apply. 


\title{
Dynamics of the spatial electron density distribution of EUV-induced plasmas
}

\author{
R M van der Horst ${ }^{1}$, J Beckers ${ }^{1}$, E A Osorio ${ }^{2}$ and V Y Banine ${ }^{1,2}$ \\ 1 Department of Applied Physics, Eindhoven University of Technology, 5600MB Eindhoven, \\ The Netherlands \\ 2 ASML The Netherlands B.V., 5500AH Veldhoven, The Netherlands \\ E-mail: r.m.v.d.horst@tue.nl
}

Received 17 August 2015, revised 1 September 2015

Accepted for publication 3 September 2015

Published 2 October 2015

\begin{abstract}
We studied the temporal evolution of the electron density distribution in a low pressure pulsed plasma induced by high energy extreme ultraviolet (EUV) photons using microwave cavity resonance spectroscopy (MCRS). In principle, MCRS only provides space averaged information about the electron density. However, we demonstrate here the possibility to obtain spatial information by combining multiple resonant modes. It is shown that EUV-induced plasmas, albeit being a rather exotic plasma, can be explained by known plasma physical laws and processes. Two stages of plasma behaviour are observed: first the electron density distribution contracts, after which it expands. It is shown that the contraction is due to cooling of the electrons. The moment when the density distribution starts to expand is related to the inertia of the ions. After tens of microseconds, the electrons reached the wall of the cavity. The speed of this expansion is dependent on the gas pressure and can be divided into two regimes. It is shown that the acoustic dominated regime the expansion speed is independent of the gas pressure and that in the diffusion dominated regime the expansion depends reciprocal on the gas pressure.
\end{abstract}

Keywords: electron density, MCRS, EUV, expansion

(Some figures may appear in colour only in the online journal)

\begin{abstract}
Nowadays more and more devices are being connected to the internet. Current predictions indicate that in 2020 more than 25 billion devices will be connected [1]. This results in an increasing global demand for more computational power and memory capacity at higher efficiency. This can be achieved by miniaturizing computer chips. From lithography point of view, for that purpose the next logical step is to use extreme ultra-violet (EUV) radiation at $13.5 \mathrm{~nm}(92 \mathrm{eV})$. These high energy photons are partially absorbed by the background gas in the lithography tool, generating a plasma due to photoionization. This EUV-induced plasma is a relatively new phenomenon, which got attention due to the research in the area of EUV lithography [2, 3]. The presence of such plasma can have a long term influence on the optical lifetime of the lithography tool and this influence is yet not fully understood. It is
\end{abstract}

essential to understand the physics of EUV-induced plasmas to predict this impact.

Currently, EUV sources and EUV-induced processes are under huge investigation, for example [4-15]. Recently, Dolgov et al presented an experimental set-up to study EUVinduced processes (e.g. carbon cleaning and ion sputtering) on multilayer mirrors [12]. Moreover, the emission of plasma induced by EUV is studied using optical emission spectroscopy in both man-made plasma [8-11] and astrophysical studies $[16,17]$. In previous work, the influence of EUV-induced plasmas on mirrors in the lithography tool was studied using numerical calculations $[3,18]$. These authors also attempted to determine the electron density with Langmuir probes, however, they concluded that these probes are not feasible [18]. In recent work we reported the first time-resolved and 

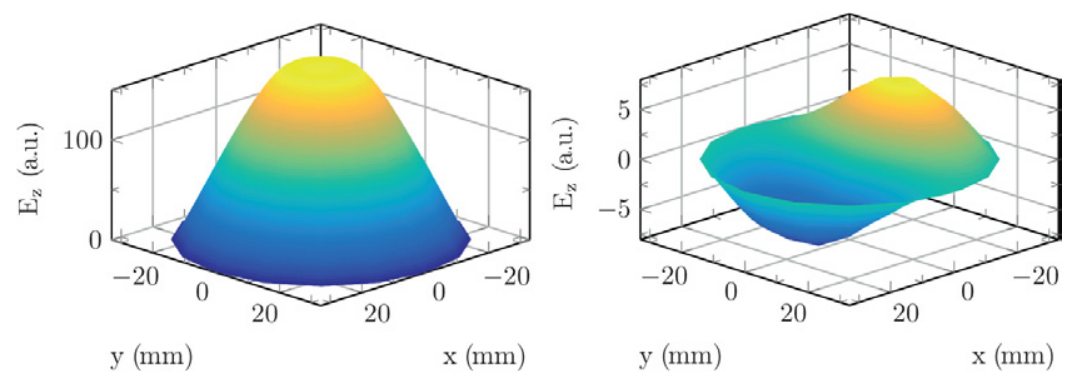

Figure 1. The electric field in the $z$-direction of $\mathrm{TM}_{010}$ (left) and $\mathrm{TM}_{110}$ mode (right). This electric field is calculated within the plasimo framework [28, 29].

non-intrusive measurements of the electron density in EUVinduced plasmas in argon by applying microwave cavity resonance spectroscopy $[19,20]$.

In this contribution we study the expansion of the electron density distribution in EUV-induced plasmas in argon as a function of the gas pressure using MCRS temporally and spatially resolved.

MCRS measurements [21, 22] have been used in other studies to determine the electron density in radio-frequency (RF) plasmas [23-25], discharge tubes [26, 27] and recently in EUV-induced plasmas [19]. Since the method is already extensive described in these publications, we suffice with a brief description of the key concepts only. In MCRS measurements a standing wave is excited in an aluminium (cylindrical) microwave cavity (in our case the cavity as an inner radius of $33 \mathrm{~mm}$ and an inner height of $20 \mathrm{~mm}$ ). The standing waves only exist at specific frequencies, the resonant frequencies $\omega_{0}$, which depend, amongst others, on the permittivity of the medium inside the cavity. When free electrons are created in the cavity, the permittivity changes, which results in a shift $\Delta \omega$ of the resonant frequency. The electron density can be calculated from this shift [23]:

$$
\bar{n}_{\mathrm{e}}=\frac{2 m_{\mathrm{e}} \epsilon_{0}}{e^{2}} \frac{\omega^{2}}{\omega_{0}} \Delta \omega
$$

where $m_{\mathrm{e}}$ is the electron mass, $\epsilon_{0}$ is the permittivity of vacuum, $e$ the elementary charge and $\omega$ and $\omega_{0}$ are the resonant frequencies with and without plasma, respectively. It should be noted that this method gives the averaged electron density weighted with the square of the local electric field $\vec{E}(\vec{r})$ of the excited resonant mode [19]:

$$
\bar{n}_{\mathrm{e}}=\frac{\int_{\text {cavity }} n_{\mathrm{e}}(\vec{r}) E^{2}(\vec{r}) \mathrm{d} \vec{r}}{\int_{\text {cavity }} E^{2}(\vec{r}) \mathrm{d} \vec{r}} .
$$

It should be noted that a single measurement does not contain spatial information about the electron density at all, nor about the expansion of the plasma. Here, we propose the usage of a second mode with a different electric field distribution, meaning that the (spatially dependent) weighting of the electron density is different. Hence, we can obtain spatial information of the electron density, i.e. information about the expansion of the electron density distribution. In our experiments we used two resonant modes, the $\mathrm{TM}_{010}$ and $\mathrm{TM}_{110}$ mode (figure 1). The $\mathrm{TM}_{010}$ mode has a maximum electric field in the centre of the cavity. The $\mathrm{TM}_{110}$ mode has a maximum electric field approximately at half the radius of the cavity (figure 1). This mode has also an azimuthal dependence, however, since the electron density distribution is cylindrically symmetric, the orientation of the mode is irrelevant. In principle any combination of resonant modes can be used, as long as the electric fields of the used modes have a significant different radial dependence.

We obtain spatial information here as follows. Assume we can write the spatial electron density distribution as:

$$
n_{\mathrm{e}}(r, t)=\tilde{n}_{\mathrm{e}} f(r, t),
$$

with $\tilde{n}_{\mathrm{e}}$ the maximum spatial density and $f(r, t)$ a radial distribution function. The electron density measured with the $\mathrm{TM}_{010}$ mode $\bar{n}_{\mathrm{e}}^{010}(t)$ is given by:

$$
\bar{n}_{\mathrm{e}}^{010}(t)=\tilde{n}_{\mathrm{e}} \frac{\int_{\text {cavity }} f(r, t) E_{010}^{2}(\vec{r}) \mathrm{d} \vec{r}}{\int_{\text {cavity }} E_{010}^{2}(\vec{r}) \mathrm{d} \vec{r}},
$$

with $E_{010}(\vec{r})$ the electric field of the $\mathrm{TM}_{010}$ mode. The electron density measured with the $\mathrm{TM}_{110}$ mode $\bar{n}_{\mathrm{e}}^{110}(t)$ is given by:

$$
\bar{n}_{\mathrm{e}}^{110}(t)=\tilde{n}_{\mathrm{e}} \frac{\int_{\text {cavity }} f(r, t) E_{110}^{2}(\vec{r}) \mathrm{d} \vec{r}}{\int_{\text {cavity }} E_{110}^{2}(\vec{r}) \mathrm{d} \vec{r}},
$$

with $E_{110}(\vec{r})$ the electric field of the $\mathrm{TM}_{110}$ mode. If we divide $\bar{n}_{\mathrm{e}}^{010}(t)$ by $\bar{n}_{\mathrm{e}}^{110}(t)$, we obtain:

$$
\frac{\bar{n}_{\mathrm{e}}^{010}(t)}{\bar{n}_{\mathrm{e}}^{110}(t)}=\frac{\int_{\text {cavity }} f(r, t) E_{010}^{2}(\vec{r}) \mathrm{d} \vec{r} \int_{\text {cavity }} E_{110}^{2}(\vec{r}) \mathrm{d} \vec{r}}{\int_{\text {cavity }} f(r, t) E_{110}^{2}(\vec{r}) \mathrm{d} \vec{r} \int_{\text {cavity }} E_{010}^{2}(\vec{r}) \mathrm{d} \vec{r}}=\Psi(t) .
$$

By dividing the weighted densities measured with the $\mathrm{TM}_{010}$ and $\mathrm{TM}_{110}$ mode, we obtain $\Psi(t)$ which contains information about $f(r, t)$. However, $f(r, t)$ still cannot be retrieved directly. This is why we need a set of distribution functions $f(r)$ which describe the general expansion of the plasma. For all these distribution functions we can calculate $\Psi$ : $\Psi_{\text {calc }}$. Now, we have created a lookup table and can determine $f(r)$ from $\Psi(t)$ at every moment in time. Hence, we have obtained information about the plasma expansion.

To obtain the set of distribution functions, we used a simplified diffusion model which solves the diffusion equation:

$$
\frac{\partial f(\vec{r}, t)}{\partial t}=D \nabla^{2} f(\vec{r}, t)
$$




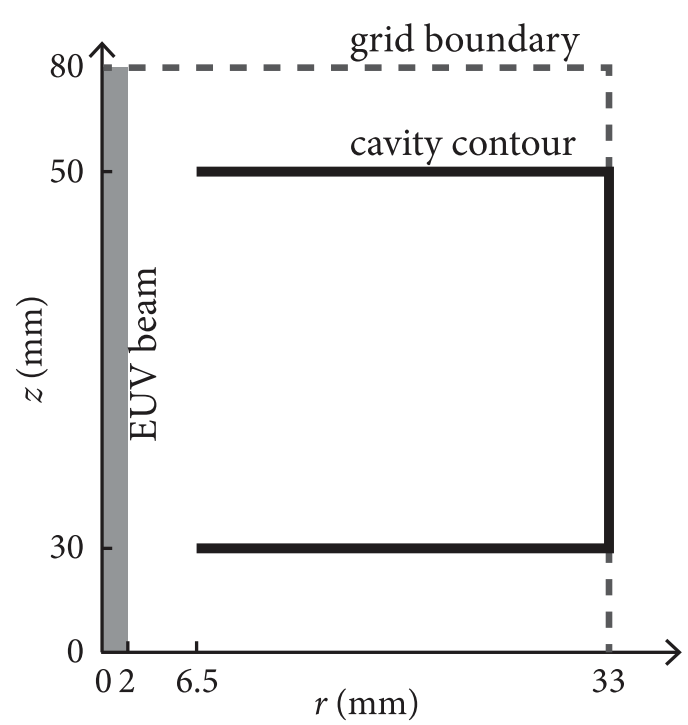

Figure 2. Schematic overview of the geometry used in the diffusion model. The boundary of the grid is spanned by the axis and the dashed lines. A Neumann boundary condition was applied on the $r=0$ axis and Dirichlet boundary conditions were applied on the other boundaries of the grid and on the walls of the cavity.

where $D$ is the diffusion coefficient. We are interested in the spatial shape of the distribution only, so the time scale and, hence, the value of $D$ is not important. This is why it is set to 1 here. It should be noted that in the above equation we assume that the electron temperature is always uniform in space, which is an oversimplification. However, it yields a good first approximation of the spatial shapes of the distribution. The cavity is axis-symmetric, so it can be represented with a 2-dimensional (radius $r$ and height $z$ ) cylindrical geometry (figure 2 ). The total computational grid has a radius of $33 \mathrm{~mm}$ and a height of $80 \mathrm{~mm}$ in order to take into account the plasma outside the cavity. A Neumann boundary condition was applied on the $r=0$ axis and Dirichlet boundary conditions on the other boundaries of the grid. On the walls of the cavity we also applied Dirichlet boundary conditions. The initial density distribution is not uniform, but, since the plasma is generated by photoionization, has a similar shape as the EUV beam. Hence, for the initial density distribution (initial condition) in the diffusion model, the shape of the EUV beam is used, which can be approximated by a Gaussian with a full width at half maximum (FWHM) of $2 \mathrm{~mm}$. The diffusion equation is solved using the linear solver in Matlab; the grid has 330 point in the $r$-direction and has 160 points in the $z$-direction, the total computational time is a few minutes on a normal desktop computer. At every time step of the simulation, we used the calculated density distribution and the calculated electric fields of the modes to determine the ratio $\Psi_{\text {calc }}$ with equation (6). Furthermore, we averaged the calculated density distribution over the height of the cavity and determined the FWHM of the resulting radial distribution. The calculated ratio $\Psi_{\text {calc }}$ as a function of the FWHM is plotted in figure 3 .

The experiment set-up consists of three different chambers (see [20] for detailed information). The source chamber contains the EUV source, which is a xenon-based discharge

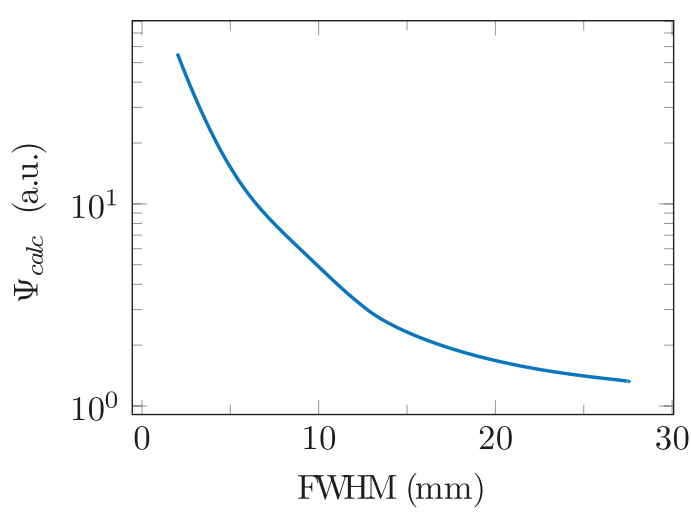

Figure 3. The calculated ratio of the electron density measured with the $\mathrm{TM}_{010}$ and $\mathrm{TM}_{110}$ mode versus the FWHM of the density distribution.

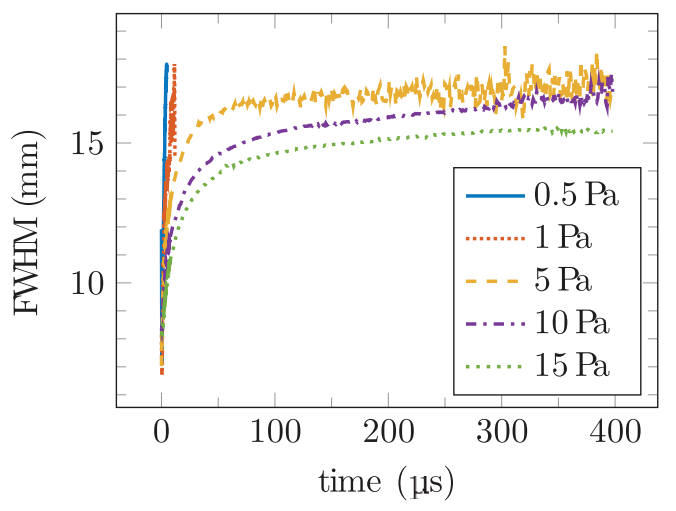

Figure 4. The evolution of the FWHM of the radial electron density distribution for various argon pressures.

produced plasma [30]. This source generates an EUV pulse with a duration of 100-200 ns, a repetition rate of $500 \mathrm{~Hz}$ and a pulse energy of $44 \mu \mathrm{J}$. A set of elliptical mirrors in the collector chamber focusses the EUV beam in the intermediate focus (IF) located in the measurement chamber. Between the collector and measurement chamber we placed a spectral purity filter (SPF) which only transmits light between 10$20 \mathrm{~nm}$. A cylindrical microwave cavity is placed around the IF. During a measurement we continuously monitor the resonant frequency of the cavity. Details of the diagnostics can be found in our previous publications [19, 20].

We measured the electron density using the $\mathrm{TM}_{010}$ and $\mathrm{TM}_{110}$ modes and determined the ratio $\Psi_{\exp }(t)$. By comparing the measured ratio with the calculated ratio in figure 3 , we obtain the FWHM as a function of time. This FWHM evolution is given in figure 4 for various argon pressures. The results show that the FWHM reaches an asymptote at approximately $17 \mathrm{~mm}$. From our diffusion model we know that this corresponds to a total width of about $63 \mathrm{~mm}$, meaning that the electrons are distributed over the entire cavity, which has a diameter of $66 \mathrm{~mm}$.

Furthermore, the results in figure 4 show that the plasma expands slower at elevated pressures. We determined the average speed of the expansion by measuring the slope of the FWHM as a function of time between $0.3-1.3 \mu \mathrm{s}$. This speed is plotted in figure 5 as a function of pressure. We can distinguish two regimes: a regime at low pressures where 


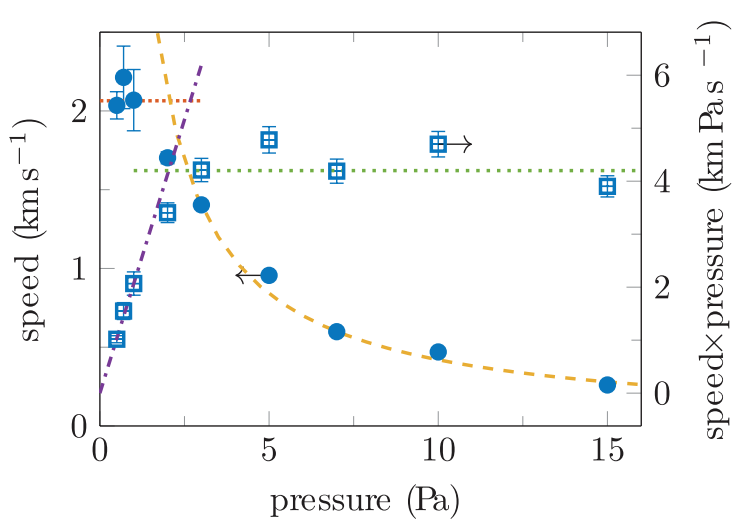

Figure 5. The speed of the expansion of electron density distribution in argon as a function of pressure $(\bullet)$. The fit in the acoustic dominated regime $(. . . \ldots .$.$) has a value$ of $(2.06 \pm 0.07) \times 10^{3} \mathrm{~m} \mathrm{~s}^{-1}$ and the fit in the diffusion dominated regime (-- ) has a proportionality constant of $(4.20 \pm 0.02) \times 10^{3} \mathrm{~m} \mathrm{~Pa} \mathrm{~s}^{-1}$. For better visibility of the two regimes, we also plotted the expansion speed times, the pressure as a function of the gas pressure $(\cdot)$ and the corresponding fits in the acoustic dominated regime $(-.-$.$) and diffusion dominate regime (\ldots \ldots \ldots)$. The arrows indicate to which axis the graphs correspond. The vertical bars indicate the error.

the expansion speed is constant and a regime at high pressures where the expansion speed depends reciprocal on the pressure. These two regimes are better visible if we plot the expansion speed times the pressure as a function of the gas pressure (figure 4). At low pressure, the mean free path of the ions is larger than the radius of the plasma. This means that the expansion speed is given by the ion acoustic speed [31]:

$$
c_{s}=\sqrt{\frac{k_{\mathrm{b}} T_{\mathrm{e}}}{m_{\mathrm{i}}}},
$$

with $k_{\mathrm{b}}$ the Boltzman constant, $T_{\mathrm{e}}$ the electron temperature and $m_{\mathrm{i}}$ the ion mass. This speed is independent of the pressure and, hence, for pressures for which the mean free path is longer than the radius, we expect a constant speed which is equal to the ion acoustic speed. We call this the acoustic dominated regime. This constant speed is what we observe below $2 \mathrm{~Pa}$. At higher pressures, the mean free path of the ions is smaller than the plasma radius. This means that the expansion speed is determined by the ambipolar diffusion coefficient: $v=D_{\mathrm{amb}} / d_{\text {diff }}$, with $d_{\text {diff }}=6 \mathrm{~mm}$ the typical diffusion length obtained from our diffusion model and $D_{\mathrm{amb}}$ the ambipolar diffusion coefficient:

$$
D_{\mathrm{amb}}=\mu_{\mathrm{i}}^{0} \frac{10^{5}}{p} \hat{T}_{\mathrm{e}}
$$

where $\mu_{\mathrm{i}}^{0}$ is the ion mobility at $10^{5} \mathrm{~Pa}, p$ is the pressure and $\hat{T}_{\mathrm{e}}$ is the electron temperature in eV [32]. Since $D_{\mathrm{amb}}$ is inversely proportional to the pressure [32], we expect a reciprocal dependence of the expansion speed on the pressure with a proportionality constant of $10^{5} \mu_{\mathrm{i}}^{0} \hat{T}_{\mathrm{e}} / d_{\text {diff }}$. This reciprocal dependence is exactly what we observe above $3 \mathrm{~Pa}$. We call the regime where the ion mean free path is smaller than the radius of the plasma the diffusion dominated regime.

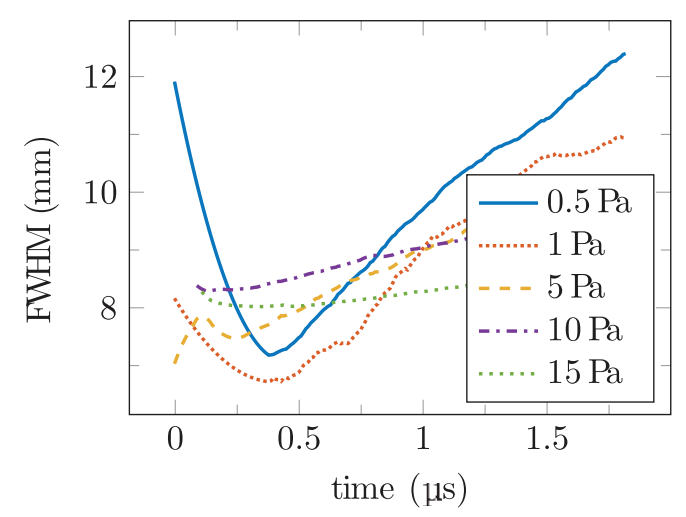

Figure 6. A zoom in at the start of the evolution of the FWHM of the radial electron density distribution for various argon pressures. The EUV pulse ends at about 120 ns.

The transition from the acoustic dominated to the diffusion dominated regime occurs when the mean free path of the ions $\left(k_{\mathrm{b}} T_{\mathrm{g}} / \sigma_{i a} p\right)$ is equal to the radius of the plasma (i.e. the ion distribution). The ion neutral collision cross section $\sigma_{i a}$ is $8 \times 10^{-19} \mathrm{~m}^{2}$ [32]. We assume that, initially, the radius of the ion distribution is similar to the radius of the EUV beam, which has a total width of $4 \mathrm{~mm}$ just after the EUV pulse [19]. This means that the transition occurs at a pressure of about 2 $\mathrm{Pa}$, which is what we observed experimentally $(2.0 \pm 0.1 \mathrm{~Pa})$.

Surprisingly, before the plasma starts to expand, it contracts (figure 6). This could be because the electrons are generated mainly in the centre of the cavity (the location of the EUV beam), i.e. the density in the centre increases faster than near the walls of the cavity. This can lead to a decrease in the FWHM of the electron density distribution, which is observed as a contraction. If this mechanism is causing the contraction, the duration of the contraction is related to the time ionization still occurs. In [20] it was shown that the ionization time is longer at elevated pressures, meaning that also the duration of the contraction should be longer at elevated pressures. Experimentally, however, the opposite is observed, meaning that this mechanism cannot explain the contraction. Another explanation for the contraction is as follows. At the very start of the EUV pulse, electrons which are created run away towards the wall leaving the positive ions behind. This results in a potential difference between the ion core and the wall of the cavity [20]. After this potential difference is established, new electrons created by the EUV beam in the centre of the cavity cannot reach the wall of the cavity due to this potential difference. As a result, these electrons will start to oscillate within the cavity, at certain moments these electrons collide with argon atoms. Due to these collisions, the electrons will lose some of there energy. Hence, the electrons can overcome less potential difference, i.e. the distance the electrons can move away from the ion core becomes smaller as a function of time. As a result, the electron density distribution contracts. The time scale of this contraction is thus related to the collision time of the electrons. This collision time is less than $20 \mathrm{~ns}$ above electron energies of $20 \mathrm{eV}$ in $0.5 \mathrm{~Pa}$ argon [33], which is much faster than the typical time scale of the contraction. However, due to the cooling of the electrons, the 


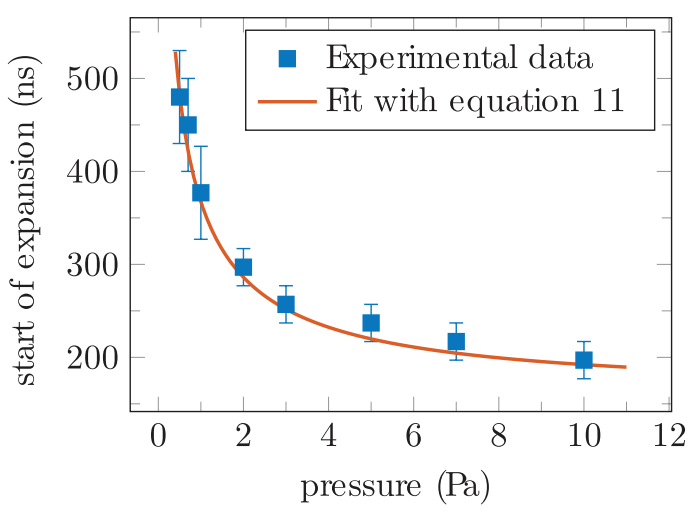

Figure 7. The time at which the electron density distribution starts to expand as a function of the gas pressure. The data is fitted with equation (11).

collision time increases to several hundreds of nanoseconds at $1 \mathrm{eV}$ [33], which is comparable to the typical time scale of the contraction.

After the contraction, the plasma starts to expand. The time at which the expansion starts is plotted in figure 7 as a function of the gas pressure. This time is related to the inertia of the ions and, hence, to the inverse of the ion plasma frequency [32]:

$$
\omega_{p, i}=\sqrt{\frac{e^{2} n_{\mathrm{i}}}{\epsilon_{0} m_{\mathrm{i}}}},
$$

with $n_{\mathrm{i}}$ the ion density and $m_{\mathrm{i}}$ the ion mass. In a previous paper [20] we showed that the electron density and, hence, the ion density, depends quadratically on the pressure. This is why the time at which the expansion starts can be fitted with:

$$
\tau_{\min }=A+\left(B p+C p^{2}\right)^{-1 / 2},
$$

where $p$ is the pressure and $A, B$ and $C$ are fitting parameters. The fit in figure 7 is in excellent agreement with the experimental data.

In conclusion, we determined the expansion of the electron density distribution in EUV-induced plasmas in argon using MCRS. It has been shown that EUV-induced plasma, although being a rather uncommon plasma, can be perfectly explained by known plasma physical laws and processes. Due to the cooling of electrons, the electron density distribution first contracts. At the moment the ions start to move, the plasma starts to expand. The speed of this expansion depends on the gas pressure and can be divided into two regimes. In the acoustic dominated regime the expansion speed does not depend on the gas pressure and in the diffusion dominated regime the plasma depends reciprocal on the gas pressure. All subsequent phenomena observed could be perfectly fitted by simplified models.

\section{Acknowledgments}

The authors would like to thank ASML for their financial support and the opportunity to use its EUV sources and J van Dijk and D Mihailova for their help with plasimo

\section{References}

[1] Terzioglu E 2014 EUV Challenges for the $7 \mathrm{~nm}$ Node in the Mobile Industry (Washington, DC: International Symposium on Extreme Ultraviolet Lithography)

[2] Banine V 2014 EUV Lithography: historical perspective and road ahead Inaugural lecture

[3] van der Velden M, Brok W, van der Mullen J, Goedheer W and Banine V 2006 Phys. Rev. E 73036406

[4] Jonkers J 2006 Plasma Sources Sci. Technol. 15 S8-S16

[5] Demmler S, Rothhardt J, Hädrich S, Krebs M, Hage A, Limpert J and Tünnermann A 2013 Opt. Lett. 385051

[6] Hädrich S et al 2014 Nat. Photon. 8 779-83

[7] Pouvesle J M et al 2003 Plasma Sources Sci. Technol. 12 S43-50

[8] Bartnik A, Fiedorowicz H and Wachulak P 2014 Phys. Plasmas 21073303

[9] Bartnik A, Wachulak P, Fiedorowicz H, Fok T, Jarocki R and Szczurek M 2014 Phys. Scr. T161 014061

[10] Bailey J E et al 2001 J. Quant. Spectrosc. Radiat. Transfer 71 157-68

[11] Cohen D H, MacFarlane J J, Bailey J E and Liedahl D A 2003 Rev. Sci. Instrum. 74 1962-5

[12] Dolgov A, Yakushev O, Abrikosov A, Snegirev E, Krivtsun V M, Lee C J and Bijkerk F 2015 Plasma Sources Sci. Technol. 24035003

[13] Gao A, Rizo P J, Zoethout E, Scaccabarozzi L, Lee C J, Banine V and Bijkerk F 2013 J. Appl. Phys. 114044313

[14] Upadhyaya M, Denbeaux G, Kadaksham A J, Jindal V, Harris-Jones J, Lee B and Goodwin F 2012 Proc. SPIE 8233 832223-6

[15] Chen J 2011 Characterization of EUV induced contamination on multilayer optics $P h D$ Thesis University of Twente Enschede

[16] Haffner L M, Reynolds R J and Tufte S L 1999 Astrophys. J. 2028

[17] Savage B D and Wakker B P 2009 Astrophys. J. 702 1472-89

[18] van der Velden M 2008 Radiation generated plasmas $P h D$ Thesis Eindhoven University of Technology Eindhoven

[19] van der Horst R M, Beckers J, Nijdam S and Kroesen G M W 2014 J. Phys. D: Appl. Phys. 47302001

[20] van der Horst R M, Beckers J, Osorio E A and Banine V Y 2015 J. Phys. D: Appl. Phys. 48285203

[21] Jackson J 1962 Classical Electrodynamics 3th edn (New York: Wiley)

[22] Rose D J and Brown S C 1952 J. Appl. Phys. 23 1028-32

[23] Haverlag M, Kroesen G M W, Bisschops T H J and de Hoog F J 1991 Plasma Chem. Plasma Process. 11 357-70

[24] Beckers J, Stoffels W W and Kroesen G M W 2009 J. Phys. D: Appl. Phys. 42155206

[25] Stoffels E, Stoffels W, Vender D, Kando M, Kroesen G and de Hoog F 1995 Phys. Rev. E 51 2425-35

[26] Gundermann S, Loffhagen D, Wagner H E and Winkler R 2001 Contrib. Plasma Phys. 41 45-60

[27] Agdur B and Enander B 1962 J. Appl. Phys. 33 575-81

[28] van Dijk J, Peerenboom K, Jimenez M, Mihailova D and van der Mullen J 2009 J. Phys. D: Appl. Phys. 42194012

[29] The Plasimo Team 2014 The PLASIMO project www.plasimo. phys.tue.nl

[30] Bergmann K, Schriever G, Rosier O, Müller M, Neff W and Lebert R 1999 Appl. Opt. 385413

[31] Bittencourt J A 2004 Fundamentals of Plasma Physics (Berlin: Springer)

[32] Lieberman M A and Lichtenberg A J 2005 Principles of Plasma Discharges and Materials Processing (New York: Wiley-Interscience)

[33] Phelps A V and Petrovic Z L 1999 Plasma Sources Sci. Technol. 8 R21-44 